\title{
ORIGINAL
}

\section{The Ultrastructural Localization of NADPH-diaphorase in the Cerebral Arteries of Rats}

\author{
Masahiro Aoyama \\ Department of Neurology, School of Medicine, Keio University, Tokyo, Japan \\ (Received for publication on May 9, 1996)
}

\begin{abstract}
The ultrastructural localization of nicotinamide adenine dinucleotide phosphate-diaphorase (NADPH-d), which has been considered to be a neuronal nitric oxide synthase (NOS), was explored in the vascular endothelial cells and perivascular nerves of the cerebral arteries in the rat. In order to detect NADPH-d activity, 2-(2'-benzothiazolyl)-5-styryl-3-(4'-phthalhydrazidyl) tetrazolium chloride was utilized as a substrate for NADPH-d histochemistry at the electron microscopic level. In vascular endothelial cells, NADPH-d positive deposits were observed on the nuclear envelope and the endoplasmic reticulum (smooth or rough surfaced). Positive deposits were seen on distinct membrane portions of the endoplasmic reticulum (ER) in the perivascular nerves (axons), but no positive materials were observed either in the cytoplasm of the endothelial cells or in the axoplasm of the perivascular nerves. It was concluded that NOS is located on the membranes of the ER and the nuclear envelope, and that NOS may play substantial roles in the regulation of the cerebral vessels. (Keio J Med 45 (4): 318-323, December 1996)
\end{abstract}

Key words: NADPH-diaphorase, nitric oxide synthase, cerebral blood vessels, electron microscope, endoplasmic reticulum

\section{Introduction}

Nitric oxide (NO) has been identified not only as an endothelium-derived relaxing factor synthesized by vascular endothelial cells, but also as a neurotransmitter both in the central and peripheral nervous system. ${ }^{1-8}$ NO is synthesized from L-arginine by nitric oxide synthase (NOS) ${ }^{5}$ Recently, neuronal nicotinamide adenine dinucleotide phosphate-diaphorase (NADPH-d) has been proven to be NOS per se and NOS-containing fibers around the cerebral blood vessels have been identified by means of NADPH-d histochemistry. ${ }^{1,9-13}$ However, it has not been clarified whether NOS is a cytosolic or "membrane-binding" enzyme. The aim of this study is to elucidate the sites of nitric oxide production at a subcellular level. In this study, by examining the ultrastructural localization of NADPH-d activity in the cerebral arteries of rats using 2-(2'-benzothiazolyl)-5-styryl-3-(4'phthalhydrazidyl) tetrazolium chloride (BSPT) as a substrate, NADPH-d positive deposits were demonstrated in the endothelial cells and in the perivascular nerves.

\section{Materials and Methods}

Adult male Sprague-Dawley rats, six to ten monthsold and weighing $200-300 \mathrm{~g}$, were deeply anesthetized with an intraperitoneal injection of pentobarbital. The perfusion was carried out through the ascending aorta with $250 \mathrm{ml}$ of $0.01 \mathrm{M}$ phosphate buffered saline ( $\mathrm{pH} 7.4$ ) followed by $300 \mathrm{ml}$ of fixative containing $4 \%$ paraformaldehyde and $1 \%$ glutaraldehyde in $0.1 \mathrm{M}$ phosphate buffer ( $\mathrm{pH} 7.4)$. After the perfusion, the brain was removed and immersed in a fixative containing $4 \%$ paraformaldehyde in $0.1 \mathrm{M}$ phosphate buffer ( $\mathrm{pH} 7.4$ ) for 2 hours at $4^{\circ} \mathrm{C}$. The internal ethmoidal artery (IEA) was removed from the brain parenchyma carefully under a light microscope. All the specimens were processed for NADPH-d histochemistry. ${ }^{13-16}$ Each specimen was briefly incubated at $37^{\circ} \mathrm{C}$ for 90 minutes in a solution containing $1.2 \mathrm{mM}$ nicotinamide adenine dinucleotide

青山正洋

Reprint requests to: Dr Masahiro Aoyama, Department of Neurology, School of Medicine, Keio University, 35 Shinanomachi, Shinjuku-ku, Tokyo 160, Japan 


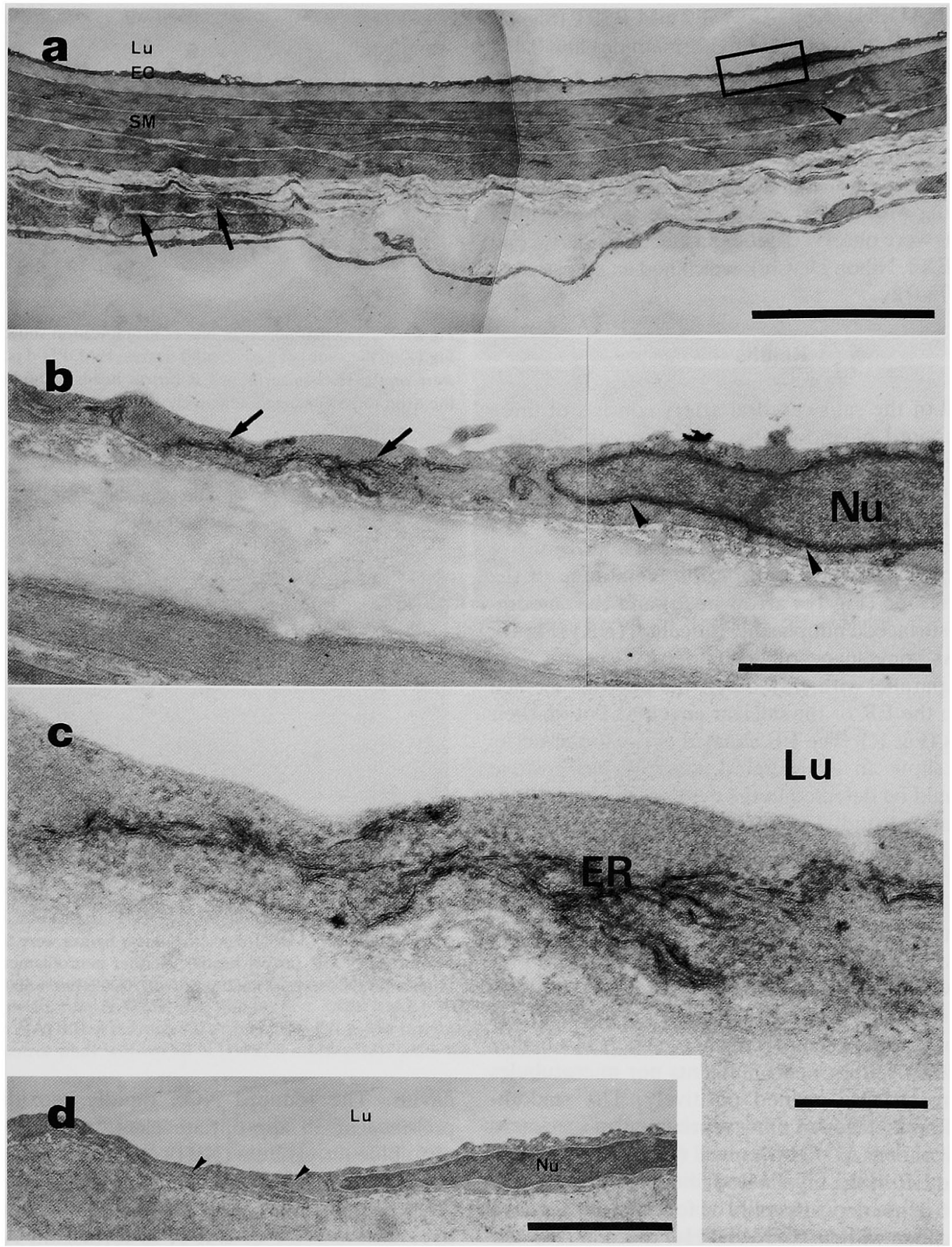

Fig 1 Electron micrographs of rat cerebral arteries with (a, b, c) and without NADPH-d reaction (d). a; Lower magnification of the rat internal ethmoidal artery $(\times 2000)$. The wall of the artery consists of three layers, endothelial cells $(E C)$, smooth muscles $(\mathrm{SM})$, and the tunica adventitia. Some varicosity of perivascular nerve fibers are in the tunica adventitia (arrows). Smooth muscles have large nuclei and some mitochondria (arrow heads). The nucleus of the endothelial cell is shown at the square. Lu; lumen of the artery, Scale $b a r=10 \mu m$. $b$; Higher magnification of the area shown by the square in a. In most of the endothelium, the positive linear deposits were observed on the membrane of the nuclear envelope (arrow head) and the smooth- and rough-surfaced endoplasmic reticulum (ER) (arrows). No positive deposits could be detected in the cytoplasm. Nu; nucleus of the endothelial cells, Scale bar $=1 \mu \mathrm{m}$. c; Higher magnification of the ER in b. Some pairs of linear dense deposits were situated in parallel with each other. The deposits seemed to be located on the membrane of the ER. Scale bar $=200 \mathrm{~nm}$. d; The control study without NADPH-d reaction showed no dense deposits in the rough-surfaced ER (arrow heads) and the nuclear envelope. Scale bar $=1 \mu \mathrm{m}$. 
phosphate (NADPH) (Kojin-Co.), 1.2 mM BSPT (Sigma, dissolved in 3-4 drops of dimethylformamide) and $0.1 \mathrm{M}$ phosphate buffer (pH8.0). Several specimens which were incubated without NADPH served as a negative control. After osmification in $2 \% \mathrm{OsO}_{4}$ (diluted in phosphate buffer $\mathrm{pH} 7.4$ ) for 4 hours and dehydration in a graded series of ethanol, the specimens were embedded in resin. After staining with uranyl acetate and lead citrate, ultra thin sections were observed under an electron microscope (JEM 1200EX2, Nihon Denshi), which had an accelerating voltage of $75.0 \mathrm{kv}$.

\section{Results}

The wall of the rat's cerebral artery consists of three layers composed of endothelial cells, smooth muscles, and the tunica adventitia (Fig 1a). In the tunica adventitia occurs some varicosity, which means the bundle of the perivascular nerve fibers (Fig 1a, arrows).

In the major portions of the endothelium, positive linear deposits were observed on the membrane of the nuclear envelope (Fig 1b, arrow head) and the smoothand rough-surfaced endoplasmic reticulum (ER) (Fig 1b, arrows). As some pairs of linear dense deposits were situated in parallel with each other, the deposits seemed not to be in the ER or the nuclear envelope but on their membranes (Fig 1c). The ER situated closer to the nuclei tended to show an exaggerated staining. No positive deposits could be detected in the cytoplasm. Figure 1d is from the control study (see Materials and Methods) and shows no dense deposits in the rough-surfaced ER and the nuclear envelope.

Some varicosity of the nerve fibers was discovered in the tunica adventitia (Fig 1a). In the axons of the perivascular nerves, the smooth-surfaced ER were presented as NADPH-d positive linear figures (Fig 2, arrow heads). The mitochondria in the axon stained negatively (Fig 2, arrow). Figure 3 shows the nerve fibers in a higher magnification. Neither neurofilaments nor microtubules (Fig 3, arrow) were stained positively. The smoothsurfaced ER in the axon of other representative specimens were positive for NADPH-d as well (Fig 4a, b, c, arrow heads). The ER in the myelinated fiber was also positive (Fig 4d). No dense deposits could be found in the negative control specimen without an NADPH-d reaction (Fig 4e).

\section{Discussion}

NOS uses arginine as its substrate, forming citrulline and NO, and it requires NADPH as an electron donor. Thus, NOS can produce NO in an NADPH-dependent fashion in response to changes in intracellular free calcium. NOS initially receives electrons from NADPH in a calcium/calmodulin- and arginine-independent step that presumably involves the reduction of the associated

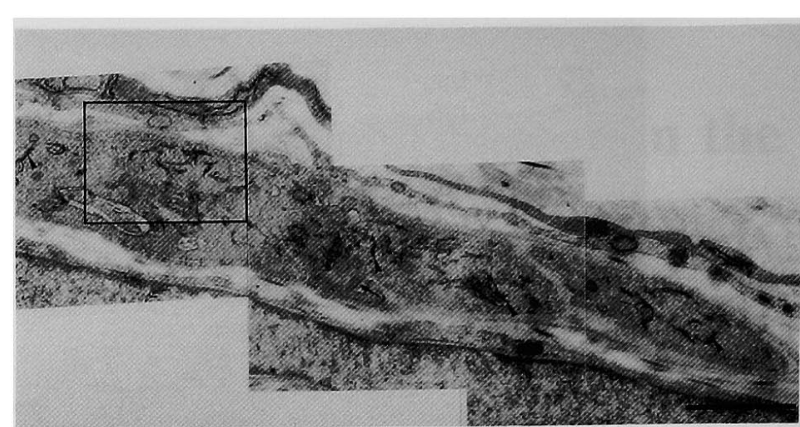

Fig 2 Perivascular nerves in higher magnification shown by arrows in Fig 1a. In the axons of perivascular nerves, NADPH-d positive deposits were on the smooth-surfaced ER (arrow heads). The mitochondria in the axon (arrow) stained negatively. Scale bar $=1 \mu \mathrm{m}$.

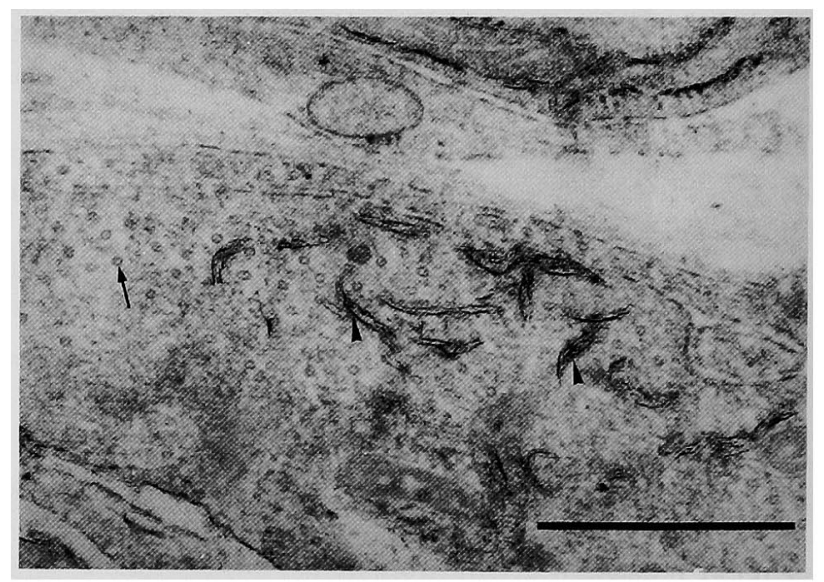

Fig 3 The nerve fibers in the higher magnification shown by the square in Fig 2. NADPH-d positive linear figures were found to be the membrane of ER (arrow heads). Neither neurofilaments nor microtubules (arrow) were stained positively. Scale bar $=500 \mathrm{~nm}$.

flavins. The reduced NOS rapidly reoxidizes in the presence of an appropriate electron acceptor, such as nitro blue tetrazolium (NBT) or BSPT, giving rise to the NADPH-d reaction product. Thus, the reduced type of NOS (a complex of NOS and flavin) is able to change a dye, such as NBT or BSPT, to formazan. Accordingly, NOS activity can be detected as the activity of NADPH-d; i.e. NADPH-d histochemistry provides a specific histochemical marker for organs producing NO. ${ }^{17}$

Some biochemical or histochemical studies have been carried out in order to investigate the subcellular localization of NOS. Biochemical studies of the endothelial cells of the bovine aorta have shown that some NOS activity could be found in the cytosolic fraction, but most of the activity was associated with the particulate fraction. ${ }^{18,19}$ Some immunohistochemical studies using 

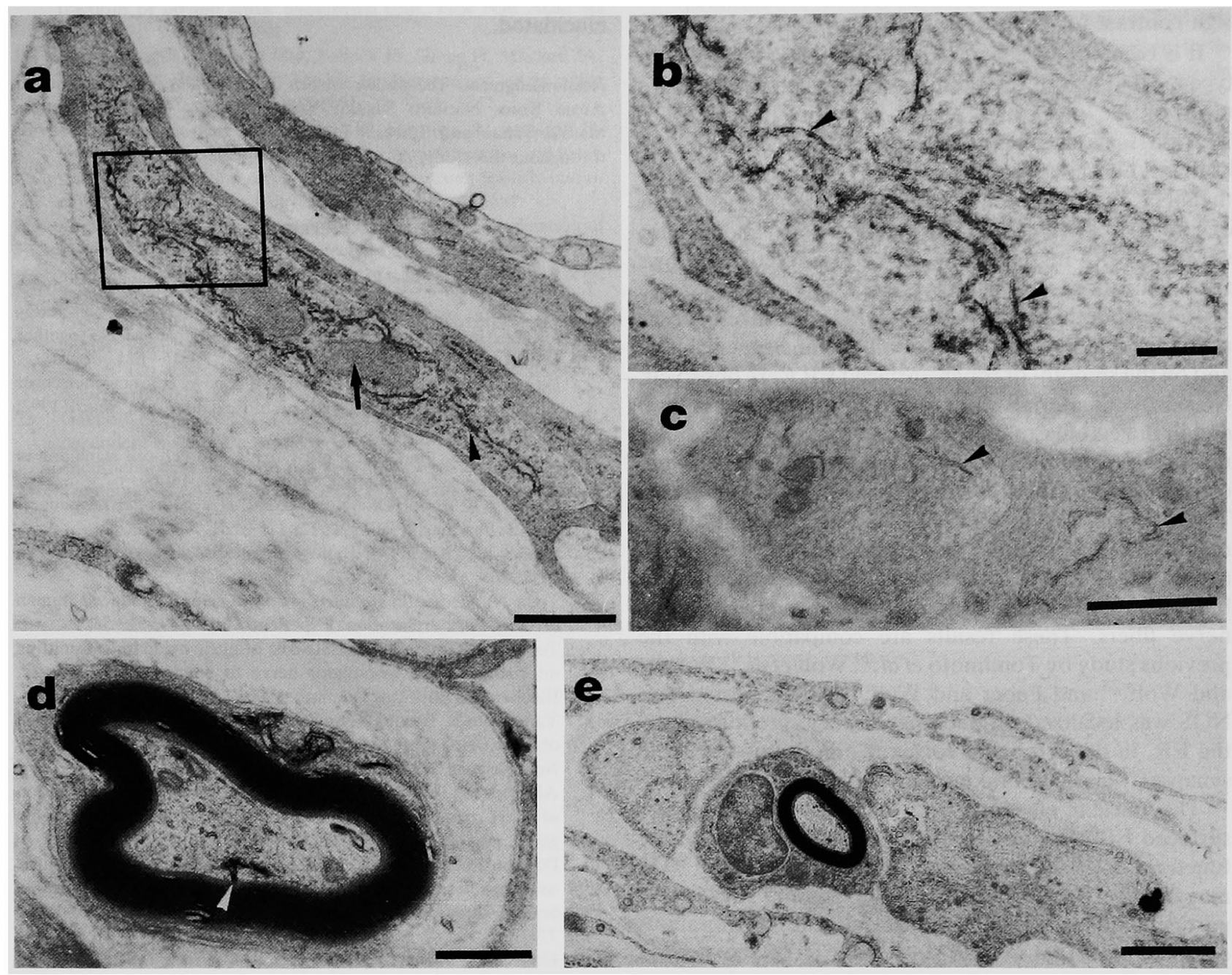

Fig 4 Electron micrographs of other representative specimens with (a, b, c, d) and without (e) NADPH-d reaction. The smooth-surfaced ERs in the axon were positive for NADPH-d as well ( $a, b, c$, arrow heads). $b$ shows the higher magnification of the square in a. Mitochondria were also negative for an NADPH-d reaction (a, arrow). The ER in the myelineted fiber was also positive (d, arrow head). No dense deposits could be found in the control study without an NADPH-d reaction (e). Scale bar $=1 \mu \mathrm{m}(\mathrm{e}), 500 \mathrm{~nm}(\mathrm{a}, \mathrm{c}, \mathrm{d}),, 100 \mathrm{~nm}(\mathrm{~b})$.

anti-NOS antibodies have proven that NOS is associated with the plasma membrane and the membrane of the cytoplasmic vesicles and is present in the cytoplasm in the endothelial cells of the human umbilical vein. ${ }^{20}$ In the endothelial cells of rabbit aorta, NOS is reported to be associated with free ribosome. ${ }^{21}$ In the rat basilar artery, NOS activity can be seen throughout the cell cytoplasm and is associated with the membrane of the mitochondria and ER in the endothelium. ${ }^{22}$ Some studies of NADPH-d histochemistry at the electron microscopic level have also been performed ${ }^{23,24}$ however the precise subcellular localization of NADPH-d (i.e. NOS), has not been fully clarified. This study clearly demonstrated the subcellular localization of NOS with NADPH-d histochemistry using BSPT in rat IEA which had been demonstrated to be denser with NADPH-d positive nerve fibers than any other main arteries in the circle of Willis at a light microscopic level by the previous study. ${ }^{17}$

NBT is a commonly utilized substrate in NADPH-d histochemistry at the light microscopic level. However, NBT is not appropriate for electron microscopic studies because the electron density of the formazan is too faint to be demonstrated. ${ }^{14,15}$ Gabbott et $a l^{23}$ employed a unique technique in the application of NBT in an electron microscopic study but failed to demonstrate NADPH-d activity directly. In this study, BSPT was utilized instead of NBT, in accordance with the method of Wolf et al ${ }^{16}$ BSPT itself does not react with osmium tetroxide but, when reduced by NOS activity, it gives off a formazan which produces a dark osmium complex and provides a 
high contrast for NOS activity. ${ }^{14}$

It is commonly recognized that NOS has several subtypes. The NOS in the brain has been reported to share most of the properties of the isoforms Ia and III. ${ }^{19}$ Isoform Ia is a constitutive enzyme purified from the brain and is called "neuronal NOS". Isoform III, endothelial NOS, is a particulate enzyme responsible for most of the NO synthesis in endothelial cells and is also constitutive. ${ }^{19}$ In this study, NADPH-d activity shows the localization of constitutive-NOS.

In the endothelial cells the NADPH-d positive deposits as an accumulation of osmium on the nuclear envelope and on the smooth- or rough-surfaced ER were observed. No positive materials were observed in the nucleoplasm and in the cytoplasm. As for the axon of the perivascular nerves, NADPH-d positive linear figures, which were considered to be the smooth-surfaced ER, were observed. The deposit was located not in the ER, but on its membrane. No positive deposits were observed in the axoplasm. It was concluded that NOS is a membranebinding protein both in the endothelium and perivascular nerve fibers. These results are compatible with the previous study by Tomimoto et al, ${ }^{24}$ Wolf et al, ${ }^{25}$ Wurdig and Wolf, ${ }^{28}$ and Faber and Wolf, ${ }^{27}$ who reported that NOS was localized on endomembranes, predominantly the ER, in the cerebellar cortex, ${ }^{26}$ hippocampal cortical synapses $^{27}$ and cerebral endothelial cells ${ }^{24}$ of the rat.

The nuclear envelope and the smooth- and roughsurfaced ER are connected with each other. One of the functions of these "membrane-binding" endothelial NOS may be the relaxation of vascular smooth muscle, or participation in the metabolism of the membranous components. The smooth-surfaced ER of the neuron is located in the cytoplasm, axoplasm and the nerve terminal, though its function has not been fully clarified. In general, the smooth-surfaced ER is considered to provide the synaptic terminals with the membrane compound in order to produce the synaptic vesicles. ${ }^{28-30}$ NOS might be transported by the smooth-surfaced ER on its membrane with a relatively slow velocity. Also the membrane of the smooth-surfaced ER changes to the synaptic vesicle at the synaptic terminals. ${ }^{28-30}$ Accordingly, it is suggested that NOS has some relationship to the endomembrane system.

In the present study, it was demonstrated that the endothelial NOS was located on the ER and on the nuclear envelope in the endothelial cells, and that neuronal NOS was located on the smooth-surfaced ER in the axon of the perivascular nerves in the rats' cerebral arteries. In conclusion, it was determined that NOS was predominantly located on the membranous structures as a membrane-binding state rather than as a cytosolic state in the cerebral arteries. The relationship of NOS and the endomembrane system as well as the function of membrane-binding NOS remains to be elucidated.

Acknowledgment: The author wishes to thank Drs Yasuo Fukuuchi, Atsuo Koto, Norihiro Suzuki, Yoko Morita, Toshihiko Shimizu, Masaki Takao and Tatsushi Fujiwara for their constructive advice throughout this study.

\section{References}

1. Bredt DS, Glatt CE, Hwang PM, Fotuhi M, Dawson TM, Snyder SH: Nitric oxide synthase protein and mRNA are discretely localized in neuronal populations of the mammalian CNS together with NADPH diaphorase. Neuron 1991; 7: 615-624

2. Dawson TM, Dawson VL, Snyder SH: A novel neuronal messenger molecule in brain: the free radical, nitric oxide. Ann Neurol 1992; 32: $297-311$

3. Garthwaite J: Glutamate, nitric oxide and cell-cell signalling in the nervous system. Trends Neurosci 1991; 14: 60-67

4. Snyder SH, Bredt DS: Nitric oxide as a neuronal messenger. Trends Pharmacol Sci 1991; 12: 125-128

5. Snyder SH, Bredt DS: Biological roles of nitric oxide. Sci Am 1992; 266: 68-71

6. Toda N: Mediation by nitrix oxide of neurally-induced human cerebral artery relaxation. Experientia 1993; 49: 51-53

7. Toda N, Okamura T: Possible role of nitric oxide in transmitting information from vasodilator nerve to cerebroarterial muscle. Biochem Biophys Res Commun 1990; 170: 308-313

8. Yamamoto R, Bredt DS, Snyder SH, Stone RA: The localization of nitric oxide synthase in the rat eye and related cranial ganglia. Neuroscience 1993; 54: 189-200

9. Afework M, Tomlinson A, Belai A, Burnstock G: Colocalization of nitrix oxide synthase and NADPH-diaphorase in rat adrenal gland. Neuroreport 1992; 3: 893-896

10. Dawson TM, Bredt DS, Fotuhi M, Hwang PM, Snyder SH: Nitric oxide synthase and neuronal NADPH diaphorase are identical in brain and peripheral tissues. Proc Natl Acad Sci USA 1991; 88: 7797-7801

11. Hope BT, Michael GJ, Knigge KM, Vincent SR: Neuronal NADPH diaphorase is a nitric oxide synthase. Proc Natl Acad Sci USA 1991; 88: 2811-2814

12. Saffrey MJ, Hassall CJ, Hoyle CH, Belai A, Moss J, Schmidt HH, Förstermann U, Murad F, Burnstock G: Colocalization of nitric oxide synthase and NADPH-diaphorase in cultured myenteric neurons. Neuroreport 1992; 3: 333-336

13. Vincent SR, Kimura H: Histochemical mapping of nitric oxide synthase in the rat brain. Neuroscience 1992; 46: 755-784

14. Kalina M, Plapinger RE, Hoshino $Y$, Seligman AM: Nonosmiophilic tetrazolium salts that yield osmiophilic, lipophobic formazans for ultrastructural localization of dehydrogenase activity. J Histochem Cytochem 1972; 20: 685-695

15. Ogawa K: Introduction to electron microscopic cytochemistry (2). Denshikenbikyo (Electron Microscopy) 1976; 11: 44-68 (in Japanese)

16. Wolf G, Wurdig S, Schunzel G: Nitric oxide synthase in rat brain is predominantly located at neuronal endoplasmic reticulum: an electron microscopic demonstration of NADPH-diaphorase activity. Neurosci Lett 1992; 147: 63-66

17. Suzuki N, Fukuuchi $Y$, Koto A, Naganuma Y, Isozumi K, Matsuoka S. Gotoh J, Shimizu T: Cerebrovascular NADPH diaphorase-containing nerve fibers in the rat. Neurosci Lett 1993; 151: $1-3$

18. Förstermann U, Pollock JS, Schmidt HH, Heller M, Murad F: Calmodulin-dependent endothelium-derived relaxing factor/nitric oxide synthase activity is present in the particulate and cytosolic 
fractions of bovine aortic endothelial cells. Proc Natl Acad Sci USA 1991; 88: 1788-1792

19. Förstermann U, Schmidt HH, Pollock JS, Sheng H, Mitchell JA, Warner TD, Nakane M, Murad F: Isoforms of nitric oxide synthase. Biochem Pharmacol 1991; 42: 1849-1857

20. Pollock JS, Nakane M, Buttery LD, Martinez A, Springall D, Polak JM, Förstermann U, Murad F: Characterization and localization of endothelial nitric oxide synthase using specific monoclonal antibodies. Am J Physiol 1993; 265: 1379-1387

21. Loesch A, Belai A, Burnstock G: Ultrastructural localization of NADPH-diaphorase and colocalization of nitric oxide synthase in endothelial cells of the rabbit aorta. Cell Tissue Res 1993; 274: $539-545$

22. Loesch A, Belai A, Burnstock G: An ultrastructural study of NADPH-diaphorase and nitric oxide synthase in the perivascular nerves and vascular endothelium of the rat basilar artery. J Neurocytol 1994; 23: 49-59

23. Gabbott PL, Bacon SJ: Histochemical localization of NADPHdependent diaphorase (nitric oxide synthase) activity in vascular endothelial cells in the rat brain. Neuroscience 1993; 57: 79-95

24. Tomimoto H, Akiguchi I, Wakita H, Nakamura S, Kimura J:
Distribution of NADPH-diaphorase in the cerebral blood vessels of rats: a histochemical study. Neurosci Lett 1993; 156: 105-108

25. Wolf G, Wurdig S, Henschke G: Nitric oxide synthase in the brain: light and electron microscopical findings based on the NADPH-diaphorase reaction. J Neural Transm Suppl 1994; 43: $105-112$

26. Wurdig S, Wolf G: Localization of NADPH-diaphorase/nitric oxide synthase activity in the rat cerebellar cortex: a light and electron microscopical study. J Hirnforsch 1994; 35: 495-499

27. Faber Zuschratter $\mathrm{H}$, Wolf $\mathrm{G}$ : Ultrastructural distribution of NADPH-diaphorase in cortical synapses. Neuroreport 1994; 5: 2029-2032

28. Broadwell RD, Cataldo AM: The neuronal endoplasmic reticulum: Its cytochemistry and contribution to the endomembrane system. J Histochem Cytochem 1983; 31: 1077-1088

29. Broadwell RD, Cataldo AM: The neuronal endoplasmic reticulum: its cytochemistry and contribution to the endomembrane system. II. Axons and terminals. J Comp Neurol 1984; 230: 231-248

30. Daikoku S: Immunohistochemistry in the research of nervous system. Igaku No Ayumi 1987; 142: 308-312 (in Japanese) 